\title{
Historia y proceso constructivo del al- tar y tabernáculo de Juan de Madrazo para la Catedral de Oviedo
}

\author{
History and construction process of the altar \\ and tabernacle of Juan de Madrazo for the \\ cathedral of Oviedo
}

\author{
MARGARITA FERnÁNDEZ MARTíNEZ \\ Arquitecto. Programa de Doctorado Patrimonio Arquitectónico: Teoría, historia, conservación \\ y restauración del patrimonio. Universidad Politécnica de Madrid
}

20800381@live.uem.es

Recibido: $17 / 11 / 2017$

Aceptado: $12 / 01 / 2018$

\section{Resumen}

La catedral de Oviedo, en el siglo XIX, sufre la reforma de la capilla mayor completada con el montaje del nuevo altar y tabernáculo en el año 1869, creado por el arquitecto Juan de Madrazo y Kuntz. En el año 1866, Madrazo comienza a proyectar el altar y tabernáculo tras haber viajado a la ciudad de Oviedo para conocer de primera mano el presbiterio y el retablo de la catedral que le proporcionó la idea para el diseño definitivo. Finalmente es en el año 1868, tras haber sido trasladadas todas las piezas que componían el altar y tabernáculo, cuando Madrazo regresa a Oviedo para hacerse cargo del envío y recibirlo en la catedral. El diseño del altar y tabernáculo recogió la influencia del arquitecto francés Viollect-le-Duc, y el gusto de este por las recreaciones de piezas medievales que servían para amueblar los edificios de estilo gótico.

Palabras clave

Restauración, altar, tabernáculo, presbiterio, memoria, intervención. 


\begin{abstract}
The cathedral of Oviedo, in the nineteenth century, undergoes the reform of the main chapel completed with the installation of the new altar and tabernacle in 1869, created by the architect Juan de Madrazo and Kuntz. In 1866, Madrazo began to project the altar and tabernacle after having traveled to the city of Oviedo to know firsthand the presbytery and the altarpiece of the cathedral that provided the idea for the final design. Finally it is in the year 1868, after having been transferred all the pieces that composed the altar and tabernacle, when Madrazo returns to Oviedo to take charge of the shipment $t$ receive it in the cathedral. The design of the altar and tabernacle included the influence of the French architect Viollect-le-Duc, and the taste of it for the recreations of medieval pieces used to furnish Gothic-style buildings.
\end{abstract}

\title{
Keywords
}

Restoration, altar, tabernacle, presbytery, memory, intervention.

Referencia normalizada: FERNÁNDEZ MARTíNEZ, MARGARITA (2018): “Historia y proceso constructivo del altar y tabernáculo de Juan de Madrazo para la Catedral de Oviedo". Arte y Ciudad. Revista de Investigación, no 13 (abril), págs. 83-102. Madrid. Grupo de Investigación Arte, Arquitectura y Comunicación en la Ciudad Contemporánea, Universidad Complutense de Madrid.

Sumario: 1. Introducción. 2. Comienzos y proyección. 3. Ejecución y diseño. 4. Montaje y viaje de Madrazo a Oviedo. 5. Traslación de la catedral al seminario. 6. Conclusiones. 7. Bibliografía.

\section{Introducción.}

En el obispado corto y de escasa trascendencia de Jose Luis Montagut Rubio (1864 - 1868), surge la reforma de la capilla mayor iniciada con el montaje de una valla que se completó con la instalación del nuevo altar y tabernáculo en el año 1869, cuya creación fue de la mano del arquitecto madrileño Juan de Madrazo y Kuntz. Hasta ese momento, en el presbiterio se encontraba ocupando la zona una mesa de altar "vulgar y ordinaría" diseñada por Justo Álvarez Amandi, y una custodia de madera dorada que también era usada en el monumento de Semana Santa. 
Es en este periodo, cuando el cabildo muestra un gran interés por dignificar en mayor medida el espacio del presbiterio, para lo cual solicitan la ayuda de Alejandro Mon y Menéndez, destacado político del momento, que había sido Ministro de Hacienda, y al que encargan que medie entre ellos y la Real Academia de Bellas Artes de San Fernando para buscar al futuro maestro que realice el nuevo diseño del altar-tabernáculo de la catedral de Oviedo. Fue con este motivo cuando Alejandro Mon se desplaza al centro madrileño en el mes de octubre de 1863 y es posible que ya en ese momento se tuviese la intuición de conceder la obra al arquitecto Juan de Madrazo. Fue el Sr. Caneda por petición de Alejandro Mon quien encargó el tabernáculo al académico Madrazo, que tras superar una enfermedad en los dos años anteriores, comenzó el proyecto en $1866^{1}$.

\section{Comienzos y proyección.}

Para comenzar la obra a pesar de tener en su posesión una buena fotografía del retablo es necesario para él, visitar dicho retablo del altar mayor para hacer un diseño y modelo que guarden la mejor relación y consonancia posible, lo que demuestra en Madrazo la necesidad de un estudio profundo tanto de la situación del entorno como del lugar de colocación del futuro elemento. Esta visita tiene lugar en el verano de 1866, donde comienza realmente el estudio y ejecución del proyecto. Es en este viaje cuando Juan de Madrazo conoce la catedral de Oviedo, al Deán y a todos los miembros del cabildo.

En este viaje obtiene la información necesaria y realiza una investigación profunda sobre la planta del presbiterio de la catedral para situar el futuro altar y tabernáculo. Es muy probable que Madrazo utilizase para su estudio el Plano del Presbiterio de la Sta. Iglesia Catedral de Oviedo, firmado por el arquitecto González con fecha 13 de agosto de 1863, plano que fue hallado en la colección privada de Madrazo y es muy probable que lo utilizase a modo de guía para el estudio y análisis de los elementos que se encontraban en el presbiterio originalmente. El plano permite tener una visión muy clara de la composición del presbiterio, el punto "a" es el presbiterio, el " $b$ " la mesa de altar solapada al punto " $\mathrm{c}$ ", donde se situaba el tabernáculo, y el punto " $\mathrm{d}$ " sería la

\footnotetext{
${ }^{1}$ Carta de Alejandro Mon, antiguo Ministro de Hacienda, dirigida a D. Juan Álvarez de la Viña en Madrid con fecha 30 de Abril de 1866, (Archivo Catedral de Oviedo - ACO).
} 
entrada al presbiterio ${ }^{2}$; los puntos representaban el retablo con una altura de 15 metros y que actualmente sigue en la catedral de Oviedo colocado del mismo modo que en esa época, y por último el punto " $\mathrm{g}$ " que representa las gradas que levantan 0.49 metros.

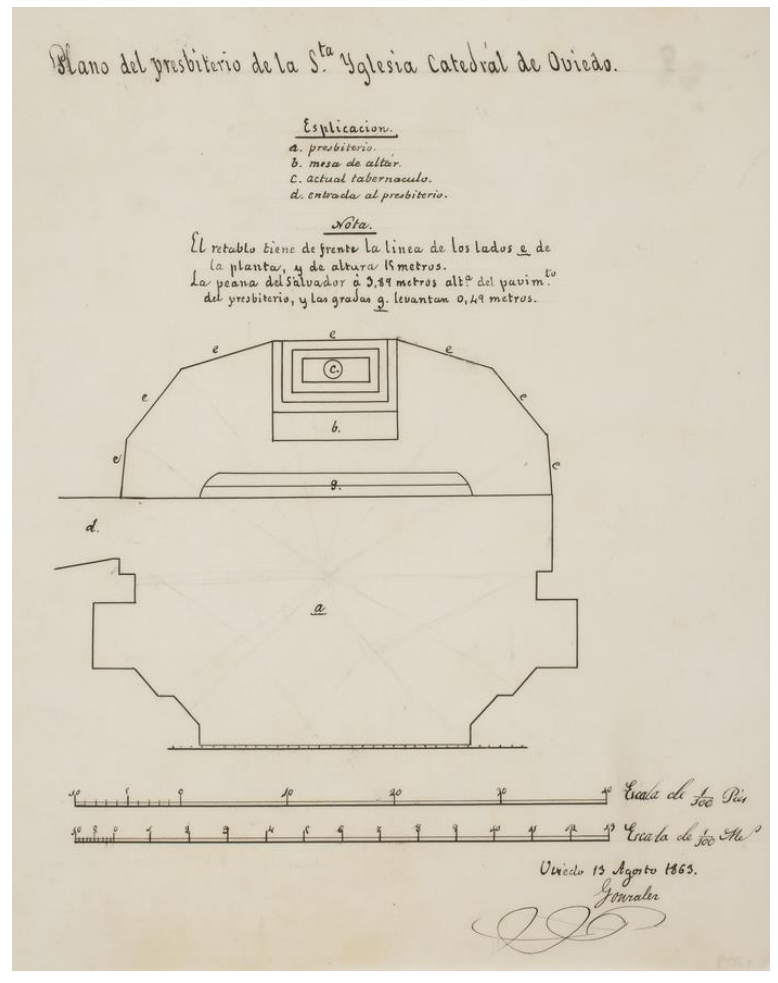

Fig. 1. Plano de la planta del presbiterio de la Sta. Iglesia catedral de Oviedo, con fecha Oviedo, 13 de agosto de 1863. Arquitecto González. Museo del Prado, D08109.

Es en Agosto de 1866, tras haber visitado la catedral de Oviedo, cuando Juan de Madrazo le hace entrega a Alejandro Mon en Madrid el modelo del tabernáculo para que este se lo remitiese a D. Juan Álvarez de la Viña, Deán de la catedral y este a su vez lo presentase al cabildo para la aprobación del proyecto, que fue trasladado hasta la ciudad de Oviedo en un cajón que guardaba el diseño, por conducto de Juan González, autor de la entrega, con un coste según la documentación de unos cuatro mil duros ${ }^{3}$.

\footnotetext{
${ }^{2}$ Es importante tener en cuenta que en esta época el sacerdote impartía la misa de espaldas al pueblo y por consiguiente el tabernáculo permanecería pegado a la pared.

${ }^{3}$ Carta n⿳0 7 de Alejandro Mon, antiguo Ministro de Hacienda, dirigida a D. Juan Álvarez de la Viña en Madrid con fecha 4 de Abril de 1866, ACO.
} 
No es hasta el 2 de Abril de 1867, cuando se hace patente la aprobación del proyecto por parte del cabildo, y es entonces cuando Madrazo puede ocuparse de la realización "sin descanso" como bien dice el propio Madrazo en una de sus cartas a Romualdo Gómez, para el éxito del proyecto.

\section{Ejecución y diseño.}

Para su realización Juan de Madrazo encarga en Madrid la parte escultórica de piedra a los escultores Víctor Mastraeten y Víctor Selen, que firman el contrato el 14 de enero de 1868, donde tenían el taller abierto en la calle del Almirante $\mathrm{n}^{\mathrm{o}}$ 19; en cambio los bronces y pedrería los encargó en un viaje que realiza a París en Semana Santa aprovechando las vacaciones de Pascua, a Fabrique d'orfêvrerie \& bronzes religieux, Alejandre Chertier, ubicada en la rue Férou $\mathrm{n}^{\mathrm{o}} 7$ près Saint Sulpice, París, y a su regreso encargó los mármoles de colores para las columnillas del altar y templete superior a las canteras de la vertiente Norte de los Pirineos, cerca de Bagneres ${ }^{4}$.

Ya es en esta fecha cuando comienzan los escultores a trabajar la piedra blanca y a esculpir talla en ella, por lo que Juan de Madrazo pide al cabildo que abone el primer plazo a los contratistas de 10.500 reales de vellón.

Para comenzar su estudio Juan de Madrazo realiza varios planos de la pre-existencia, con los cuáles va tomando diferentes decisiones para situar el altar-tabernáculo, tomando como punto de referencia el encuentro de todas las directrices de las columnas del retablo, que confluían en el centro del altar.

El retablo se muestra como una envolvente del presbiterio, en el centro de intersección coloca el altar e inmediatamente detrás el tabernáculo, condicionado este por el altar, debido a este lugar que se busca la pieza en su conjunto se muestra permeable, y como se comprueba en las fotografías antiguas en las que se encuentra el altar-tabernáculo ocupando el lugar que le corresponde en el presbiterio, este en ningún caso obstruye la visión del retablo. La idea originaria de Juan de Madrazo para saber si sería posible la situación del altar

\footnotetext{
${ }^{4}$ Carta $n^{\mathrm{o}}$ 11, de Juan de Madrazo, arquitecto, dirigida a Romualdo Gómez en Madrid con fecha 2 de Abril de 1867, en esta carta Juan de Madrazo acredita la aceptación del cabildo para realizar el proyecto de este, y también expone que envía junto a la carta "unas cuantas fotografías" para que se las dé a miembros del cabildo. Las fotografías no se han encontrado en ninguno de los archivos consultados, lo que hace creer que se han perdido dichas fotografías.
} 
mayor y tabernáculo en el punto " $\mathrm{b}$ ", tal y como se muestra en el plano, lo primero que desea es eliminar las graderías que se encontrarían debajo de la futura colocación y retirarlas hacia el punto " $\mathrm{g}$ ", entre las columnas de la catedral que cierran el presbiterio, y por último suprimir la puerta abierta en el lado izquierdo y abrir una nueva apertura bajo el retablo justo en el medio del punto " $\mathrm{d}$ ", en donde habría la necesidad también de colocar unas nuevas gradas para la subida al presbiterio y que fuese por esta entrada por donde los presbíteros podrían entrar hacia el presbiterio.
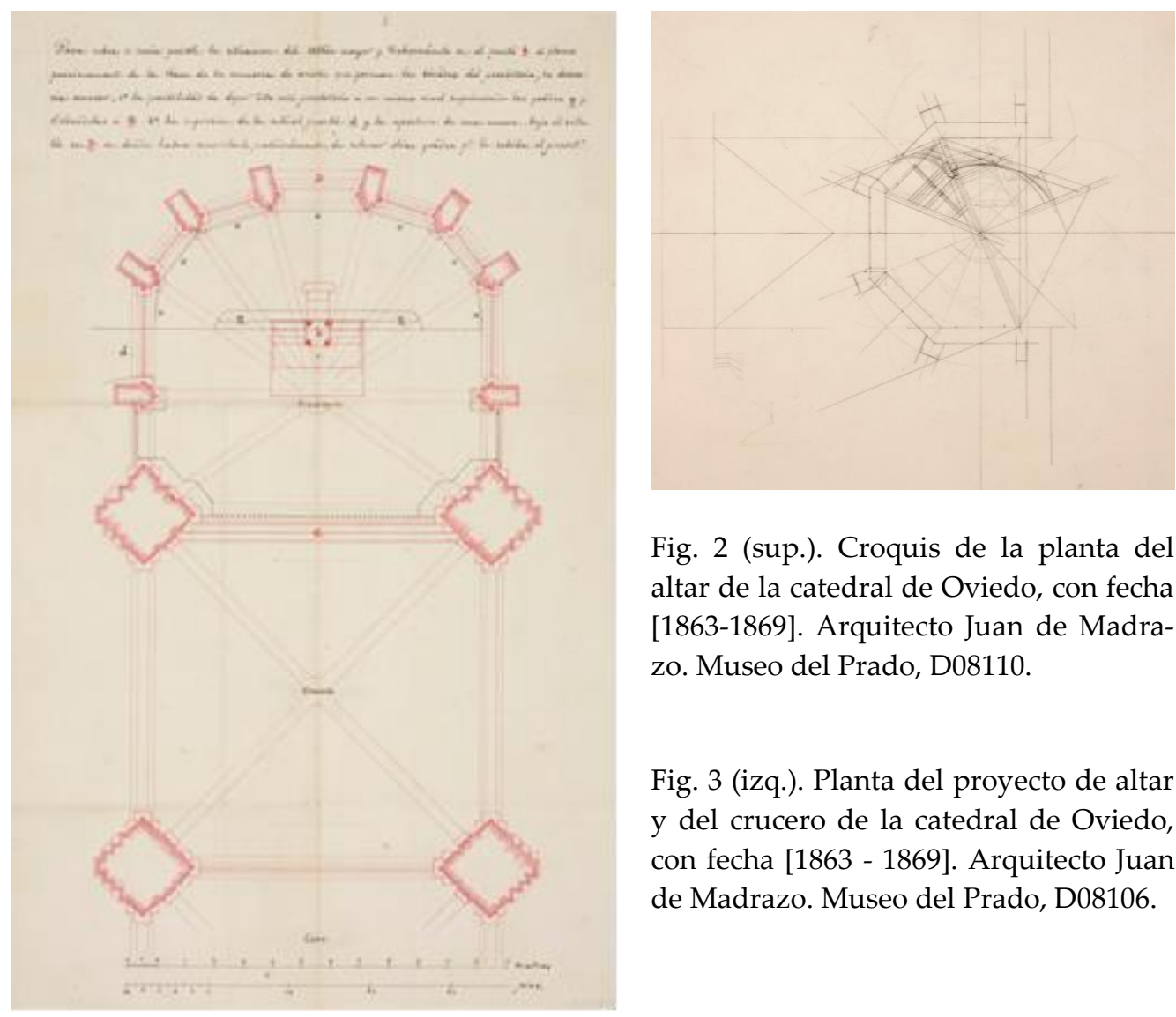

Fig. 2 (sup.). Croquis de la planta del altar de la catedral de Oviedo, con fecha [1863-1869]. Arquitecto Juan de Madrazo. Museo del Prado, D08110.

Fig. 3 (izq.). Planta del proyecto de altar y del crucero de la catedral de Oviedo, con fecha [1863 - 1869]. Arquitecto Juan de Madrazo. Museo del Prado, D08106.

Para comenzar el estudio del futuro altar-tabernáculo realiza un par de bocetos sobre el diseño del proyecto, como mínimo, ambos presentan las mismas partes, variando de forma sutil exclusivamente los detalles ornamentales, siguiendo, no obstante, con los detalles de mocárabes y las columnas, como se 
comprueba en el boceto [Fig. 4]; al final se decanta por el altar-tabernáculo que aparece en el plano-boceto [Fig. 5], ambos planos no están terminados pero al mismo tiempo se observan los diferentes elementos que componían el altar-tabernáculo.

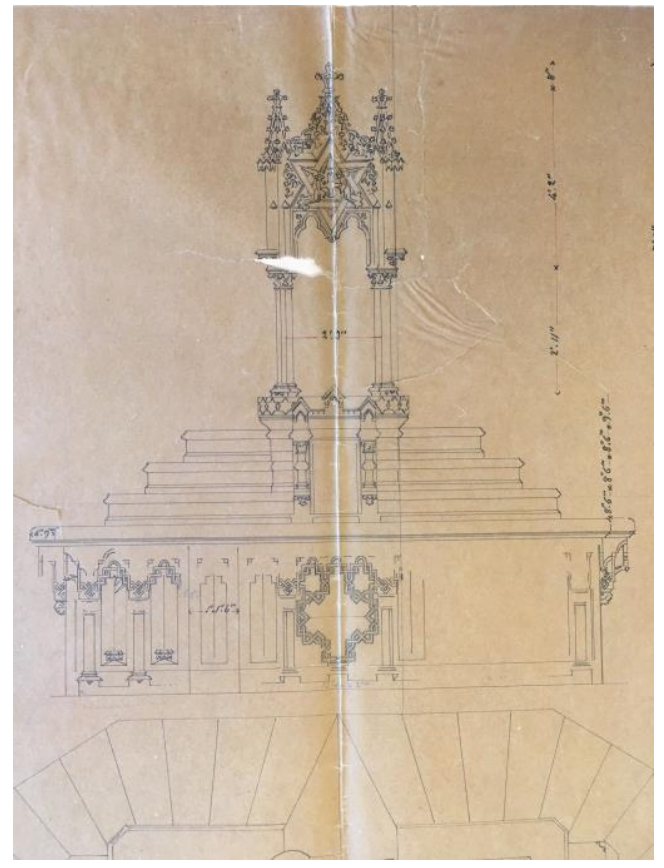

Fig. 4.- Calco del tabernáculo para el altar de la catedral de Oviedo con cotas, con fecha [1863 - 1869]. Arquitecto Juan de Madrazo. Museo del Prado, D08132.

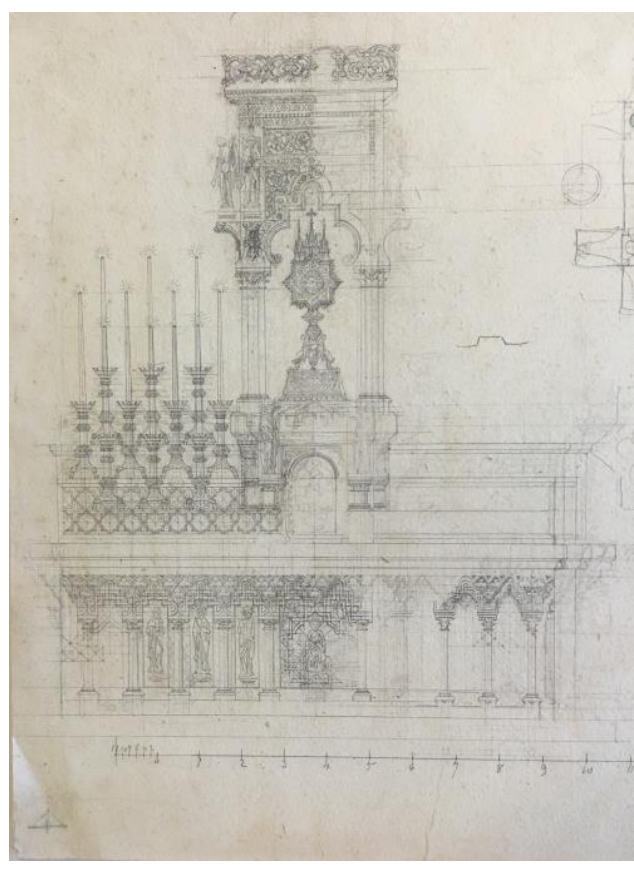

Fig. 5.- Alzado del tabernáculo de la catedral de Oviedo, con fecha [1863 - 1869]. Arquitecto Juan de Madrazo. Museo del Prado, D09021.

El diseño de Madrazo constaba de tres partes diferenciadas: la mesa de altar, el sagrario con escalinata y por último el templete.

La mesa de altar tiene unas dimensiones de $103 \times 365 \times 140 \mathrm{~cm}$, al frente de la mesa se encuentra presidiendo un relieve del Salvador entronizado, en actitud bendicente y rodeado por el Tetramorfos. Este Salvador aparece también colocado presidiendo el retablo en actitud bendicente pero al contrario que en el altar donde aparece con unas tablas, lo que aparece variado es el tetramorfos, lo cual es una representación iconográfica de un conjunto formado por cuatro elementos, esta escena cristiana se remonta al Antiguo Testamento, 
cuando el profeta Ezequiel describió en una de sus visiones cuatro criaturas que se asocian con los cuatro evangelistas.

En el altar se muestra en la parte superior izquierda, el hombre que asocia a San Mateo, a la derecha el águila que se asocia a la figura de San Juan, en la parte inferior izquierda el león que se identifica con San Marcos y por último, en la derecha el toro que se asocia a San Lucas, la diferencia con el retablo es la alteración del orden de las figuras superiores, a la izquierda aparece el águila y a la derecha el hombre. Esto documenta en parte las semejanzas con el retablo y la investigación que Madrazo realiza. A ambos lados del Salvador y en los flancos del altar se disponen las doce figuras del apostolado compuesto por pequeñas esculturas de bulto redondo de los discípulos, estas figuras se muestran erguidas de cuerpo entero y portando alguno de sus atributos característicos que los distinguen. Son doce figuras pequeñas de $55 \mathrm{~cm}$ de altura cada una. Como es tradición, la imagen de Cristo está custodiada por las figuras de San Pedro a la izquierda, portando las llaves y San Pablo a la derecha portando la espada, estos personajes aparecen como el resto de discípulos vestidos de modo antiguo, con vestiduras de la época en actitud serena.

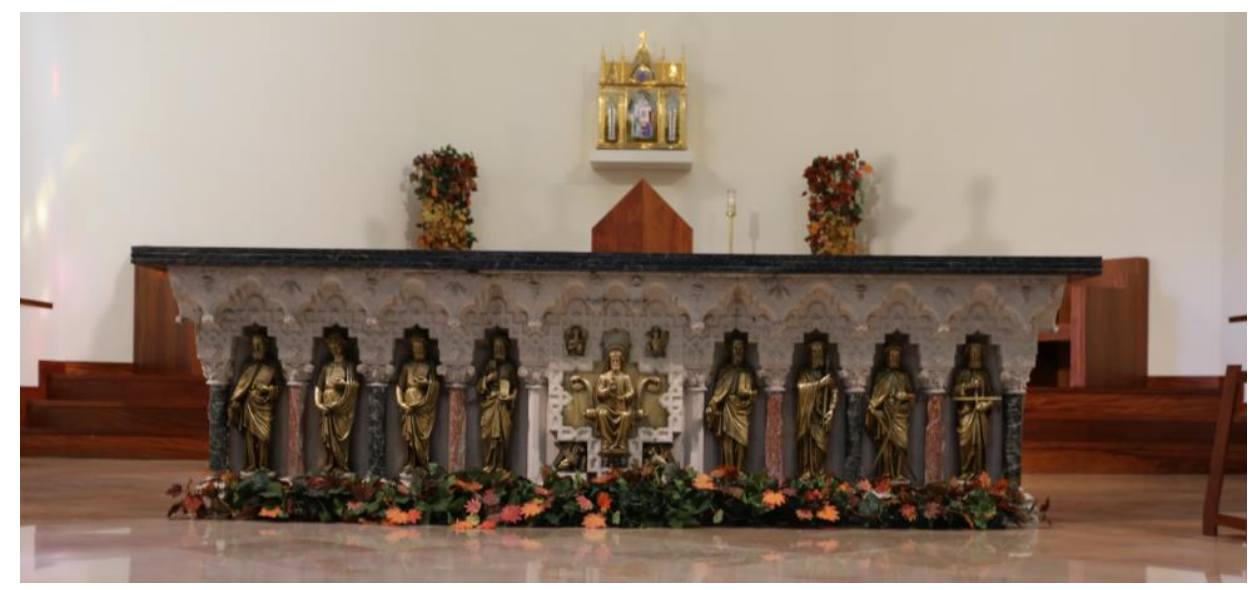

Fig. 6. Vista general del altar ubicado en la capilla mayor del seminario (Foto: Margarita Fernández Martínez, junio 2016).

Cada uno de estos apóstoles está ubicado en un marco geométrico de inspiración islámica, para realzar la figura del apóstol esta se enmarca en una especie de nicho donde se empotra la figura del apóstol, para realzar este marco geométrico aparecen en la parte superior a modo de coronación de cada após- 
tol una composición de arcos trilobulados de estilo goticista que reposan sobre pequeñas columnas cuyos fustes destacan por el cambio de material a mármol y variar los tonos entre verde y rosado, dispuesto de forma alternativa.

Para realizar la composición de arcos trilobulados Madrazo, realiza diferentes composiciones de bocetos entre los que destacan los apuntes decorativos de una cúpula de mocárabe, estos bocetos los realiza en un papel cuadriculado, siguiendo una retícula perfecta lo que le ayuda a plasmar las diferentes composiciones. Para la composición realiza un análisis matemático profundo, en primer lugar mediante el Teorema de Pitágoras analiza las alturas y mediante estas alturas es capaz de trazar las curvas que unidas entre sí formarán la cúpula mocárabe.

Coronando los fustes se encuentran los capiteles de estilo jónico, compuestos por dos volutas y en el medio de estas un detalle decorativo vegetal a modo de hojas de estilo geométrico, encima de los capiteles se encuentra el plano superior, más conocido como el ábaco, que forma el remate superior del pilar para aumentar su resistencia al esfuerzo cortante, en este caso moldeado a modo de dibujo con forma de "sierra", triángulos equiláteros seguidos para conformar todo el ábaco. La parte superior realza el nicho de inspiración islámica, este está diseñado con una cenefa geométrica, para llegar al diseño final Madrazo realiza una serie variada de diseños como remates decorativos, y como remate y diseño final coloca un motivo de flor de lys vista en planta, seguramente alguno de estos diseños los tuvo en cuenta para tomar la decisión final en el altar mayor.

Fig. 7. Apuntes para el diseño de una cúpula de mocárabes, con fecha [1863 - 1869]. Arquitecto Juan de Madrazo. Museo del Prado, D08160. 
Para las diversas cúpulas de estilo mocárabe que se encuentran tanto en la mesa de altar como en el templete, realiza diferentes tipologías con las cuáles hace las distintas composiciones como se muestra en el apunte (fig. 7). Rematando estas cúpulas mocárabes Madrazo no deja ningún espacio sin trabajar y en las zonas intermedias entre los mocárabes introduce alternativamente a modo de adornos las espigas de trigo y al lado contrario los racimos de uvas, todos ellos rematados al rededor con una talla plana de hojas vegetales de los elementos antes mencionados; con estos adornos Madrazo hace una clara alusión al momento culmen de la eucaristía, la consagración de las especies el pan y el vino.

La segunda parte de la que consta el tabernáculo es el sagrario con escalinata, sobre la mesa se coloca esta estructura escalonada que se superponía al sagrario y al mismo tiempo servía como soporte para la iluminación con velones. Esta segunda parte se encontraba retranqueada respecto a la mesa de altar hacia atrás, y en esta parte central se encuentra el sagrario custodiado por dos ángeles guardianes, y a continuación la escalinata, la cual consta de tres peldaños tanto a la izquierda como a la derecha del sagrario. Dicha escalinata está labrada por unos detalles de estilo goticista en los cuáles se han colocado unos vidrios de colores azules y rojos en los que a su vez está impreso un dibujo dorado de espigas de trigo, algo muy parecido a lo que nos encontramos en la mesa de altar, siguiendo el mismo estilo y simbología. Y entre los ángeles guardianes, de $55 \mathrm{~cm}$ de altura, se encuentra el sagrario. El sagrario tiene forma rectangular aunque el elemento central tiene forma apuntada lo que hace que la puerta sea más ligera y esbelta. La puerta de este sagrario esta labrada toda ella, sin dejar el más mínimo detalle a la imaginación, en el medio se encuentra la cruz cristiana, el principal símbolo del cristianismo, conformada por una línea vertical atravesada en su parte superior por una línea horizontal, estas están diseñadas en sus extremos por motivos decorativos vegetales y en sus brazos toda la cruz está cuajada por pedrería de color rojo, azul y verde en el empalme de las dos líneas, en el medio se encuentra el símbolo del Agnus Dei (Cordero de Dios), que se refiere a Jesucristo como víctima ofrecida en sacrificio por los pecados de los hombres, esto hace referencia al cordero que era sacrificado y consumido por los judíos durante la conmemoración anual de la Pascua. Este mismo símbolo aparece también en la mano de la figura de San Juan en la mesa del altar. Alrededor de esta figura se encuentra rodeando este elemento central de forma circular pedrería azul; esta cruz está realizada en relieve y al fondo 
toda la puerta está labrada con motivos decorativos vegetales, realizados con formas circulares y simétricas entre la parte izquierda y derecha. En las esquinas del sagrario superiores se vuelven a encontrar estos mismos detalles decorativos vegetales y es muy curioso que en la parte trasera del dibujo en las esquinas superiores se encuentran también detalles decorativos vegetales pero esta vez, impresos con un relieve mínimo cuya visión solo se observa desde el interior del sagrario, por lo que es de obviar que no se debería ver estos elementos con el altar-tabernáculo montado. La puerta se abre mediante dos bisagras y es en este elemento donde aparece la firma de los escultores que realizaron el trabajo de orfebrería A. Chertier Orfêvre à Paris.

Por último el templete, del que solo se conservan las cuatro columnas que sostienen el tabernáculo, y sobre estas se encuentran cuatro ángeles músicos. En cuanto a la custodia, que se utiliza para la exposición del santísimo no se tiene idea de en qué lugar puede estar, se puede intuir por el dibujo que realizó Juan de Madrazo de cómo podría ser, a los pies de la custodia cuatro ángeles sentados en actitud de

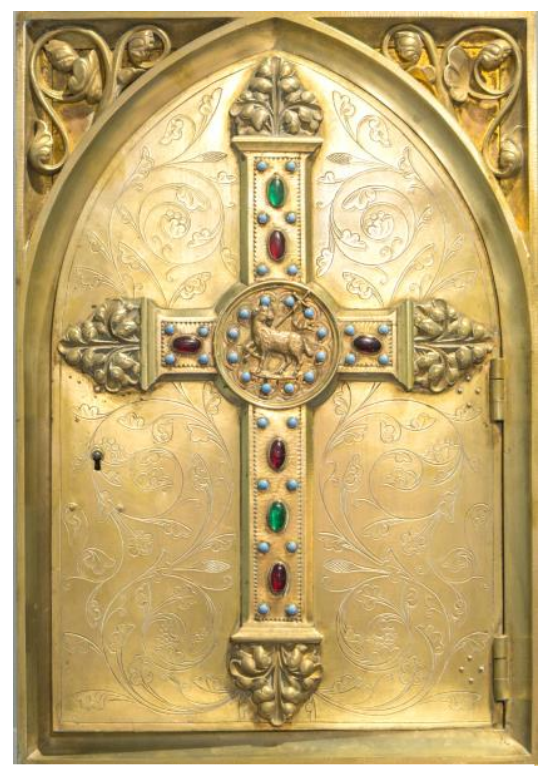

Fig. 8. Vista de la puerta del sagrario ubicado en el despacho del rector del seminario. Foto: Margarita Fernández Martínez, junio 2016. oración, en la parte central una estrella con ocho puntas decorada a su alrededor con una tipología de volutas que envuelven la parte más importante de la custodia y encima de esta un dibujo arquitectónico que parece como las torres de una catedral de estilo gótico. Coronando la custodia se vuelven a encontrar la cúpula mocárabe y todo ello remarcado con detalles decorativos vegetales, los mismos que se encuentran en el sagrario y mesa de altar, lo que crea un conjunto armónico y unitario. Para proceder a la ejecución del conjunto de altar y tabernáculo, en un principio se realizan los dibujos a tamaño natural para la ejecución de los modelos de yeso necesarios antes de trabajar en la piedra ${ }^{5}$.

\footnotetext{
${ }^{5}$ Este proceso de ejecución del proyecto se describe en la carta no 104 que escribe el propio Juan de Madrazo al Deán Juan Álvarez de la Viña.
} 
El material con el que se realiza es piedra de Guadalin, y es en Semana Santa de 1868 cuando aprovechando las vacaciones de Pascua, Juan de Madrazo viaja a París, en donde, en vista de los plenos poderes que el cabildo le confió aunque

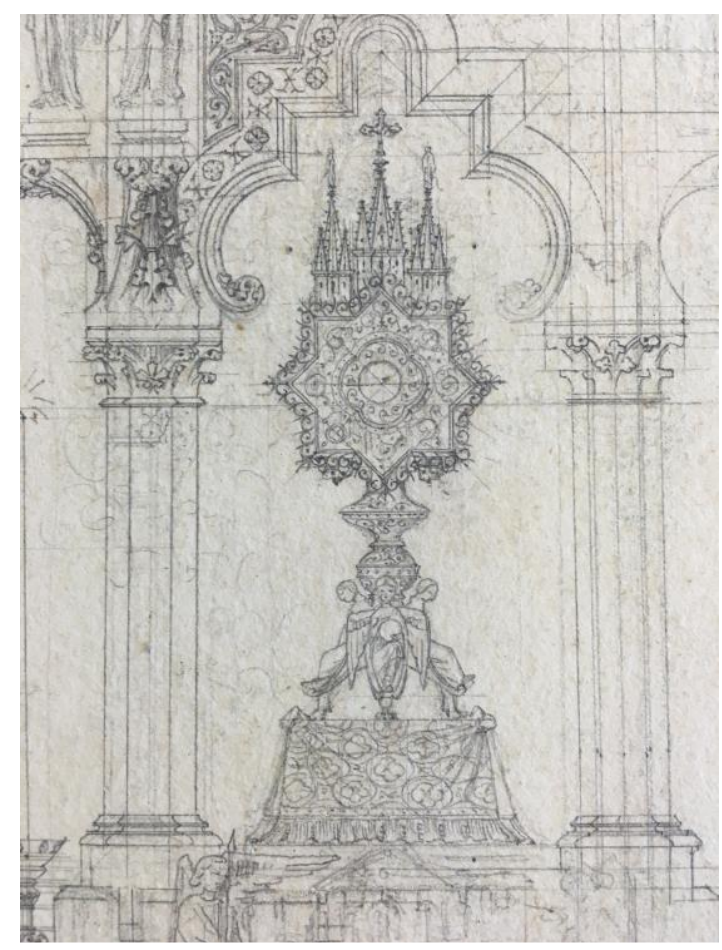

Fig. 9.- Detalle alzado del tabernáculo de la catedral de Oviedo [1863 - 1869]. Arquitecto Juan de Madrazo. Museo del Prado, D09021. Alejandro Mon (Deán de la Catedral), no se encontraba, encargó definitivamente toda la parte de bronces y pedrería de la que forma parte la estatuaria, el Salvador acompañado del Tetramorfos, el apostolado, los ángeles guardianes, los ángeles músicos y la puerta del sagrario. Y por último, encargó a las canteras de la vertiente norte de los Pirineos, cerca de Bagneres los fustes de mármol de colores rojo y verde para las columnillas del altar y templete superior.

Es en el pliego de condiciones donde queda estipulado qué han de hacer los escultores y cuál es su trabajo, así como el de los orfebres en París, que se resumiría en la realización de todas las piezas de bronces y mandarlas hasta Oviedo para la ejecución del tabernáculo y altar. En cambio, los escultores Víctor Mastraeten y Víctor Selen se obligaron ejecutar de todo coste el altar mayor y tabernáculo para la catedral de Oviedo con las siguientes condiciones: en primer lugar ejecutar tanto la mesa de altar y cuerpos superiores de Sagrario y tabernáculo el cual como se ha dicho anteriormente estaba realizado en piedra procedente de las canteras de Guadalia, y esta ha de presentar según se describe en el propio pliego de condiciones "la necesaria dureza, consistencia y firmeza para el buen resultado del ornato y que esté limpia y exenta de pelos, grietas y otros cualesquiera defectos"; con ello también se encargarían de los mármoles de colores para los fustes de columnillas que se ubicarían en los compartimentos en donde va el apostolado y 
las cuatro que sostienen el templete del tabernáculo. La segunda condición, una vez realizado el ornato y haber ejecutado los modelos preparatorios, sería la conclusión de la obra ejecutada, lo que incluiría el desplazamiento de estos hasta la capital de Oviedo, así como la colocación y armado en el sitio que le corresponde en el presbiterio y al final la conclusión total con todos los accesorios de pintura, dorados y todo lo que debería obtener para su resultado final, hasta dejar todo el tabernáculo y altar completamente concluido y en disposición de servir para el culto. En tercer lugar, que todo lo que se refiere al lugar de ubicación y la transformación que necesitaría el presbiterio en sus gradas o peldaños, como se vio en los planos primeros donde Madrazo estudia el presbiterio a fondo, será obligación del cabildo y lo ejecutarían por su propia cuenta. La cuarta condición sería la colocación de las estatuas de bronce, fijarlas y asegurarlas a las piedras, siendo de cuenta de los escultores los pernos de bronce que necesiten; tendrían por lo tanto que incrustar en su sitio asegurándolas con los necesarios mastiques cuarenta y dos piedras de imitación en la cornisa del sagrario y ciento cuarenta y cuatro en las caras del tabernáculo, haciendo los escultores los modelos de madera de peral, del modelo para la puerta de bronce del sagrario. La quinta condición, también los escultores se encargarían del andamiaje necesario para la elevación y asiento de las piezas, así como también de las máquinas y cuerdas necesarias, pero a la vez sería por cuenta del cabildo cerrar el presbiterio como lo que procediese, y después volver a abrirlo al público una vez que el altar-tabernáculo esté ya terminado.

El coste total de la obra fue de cuarenta y dos mil reales de vellón, lo que en aquella época era una cantidad muy importante de dinero. En un principio se fijó como fecha de entrega septiembre de 1868 pero esta obra como explica Madrazo en su carta fechada en Madrid el 21 de agosto de 18686, "nosotros los ar-

\footnotetext{
${ }^{6}$ Carta no 104 de Juan de Madrazo dirigida a Juan Álvarez de la Viña (Deán de la catedral). Esta carta explica el porqué de la obra finaliza con un retraso de dos meses, así como la disposición necesaria de las piezas. Es una carta muy importante ya que demuestra la actitud de amistad entre el Deán y el arquitecto. En ella queda constancia de que el Sr. Obispo de Oviedo visitó el 19 de agosto del mismo año la obra del nuevo altar mayor de la catedral y que examinó minuciosamente todos los trabajos quedándose muy satisfecho. A primeros de mes vieron también los trabajos dos señores canónigos de la catedral de León que fueron hasta Oviedo para gestionar asuntos referentes a la restauración de la catedral de León. Por lo que queda también patente algo importante ya que por estas fechas los canónigos de León andan ya detrás del arquitecto Juan de Madrazo, tras haber dicho este que no se hacía cargo de la catedral de León en febrero de 1868.
} 
quitectos ni tenemos experiencia de ellas, ni hay en realidad muchos operarios de quienes poder echar mano para ejecutarlas"; debido a esta cuestión la obra finalmente se terminó en el mes de noviembre, aunque tanto el arquitecto como el cabildo de la catedral hubiese deseado inaugurarla el día del Salvador.

El pago se realizó en cuatro plazos de diez mil y quinientos reales cada uno y se fueron recibiendo a medida que la obra iba avanzando, en Madrid recibieron los dos primeros y en la ciudad de Oviedo se efectuaron los dos restantes. El primer plazo se realizó después de ejecutar los modelos previos de escayola y con la piedra ya en el taller en disposición de comenzar la talla, el segundo cuando toda la obra ejecutada se encuentra embalada y dispuesta para el viaje a Oviedo, el tercer plazo cuando las piezas ya se encontraban desempaquetadas y en la ciudad de recepción y por último, el cuarto plazo cuando ya se dio la obra por terminada.

\section{Montaje y viaje de Madrazo a Oviedo.}

Una vez empaquetado el primer envió, Madrazo, acompañado de su mujer Margarita Tewart, se dispone a viajar a Oviedo con la finalidad de recibirlo allí a primeros del mes de septiembre, y así prepararía todos los trabajos de colocación y dirigir in situ el resto de la obra hasta su conclusión definitiva. Llegando Madrazo a Oviedo, se instala en la Fonda de Manteola donde permanece cerca de dos meses. En entonces cuando Juan de Madrazo escribe frecuentemente a su hermano Federico, con el fin de tener noticias de Madrid, y así en ellas se perciben los comentarios de la época sobre la política del momento, y en la carta número 25 donde le pide a su hermano que se encontraba en Madrid que le pagase dos mil reales a uno de los escultores que estaban trabajando en el altar en las piezas que todavía no se habían trasladado a Oviedo, pone de manifiesto el fuerte vínculo entre los dos hermanos. Mientras Madrazo permanece en Oviedo, Frassinelli ${ }^{7}$ visita las obras del altar-tabernáculo y se queda alojado con Madrazo y su mujer en la Fonda de Manteola ${ }^{8}$, permaneciendo solo días, pero ya es a principios de noviembre cuando la obra está prácticamente terminada.

\footnotetext{
${ }^{7}$ Frassinelli y Burnitz era un dibujante, bibliófilo, anticuario, naturalista y arqueólogo de origen alemán afincado en Corao, Cangas de Onís. Su mayor interés eran las iglesias medievales.

${ }^{8}$ Carta no 26 de Juan de Madrazo dirigida a su hermano Federico, en la cual escribe acerca de Frassinelli, así como de la situación en la que se encuentra Federico y al haber sido informado Juan de que este ya no se encontraba al frente de la dirección del Museo del Prado.
} 


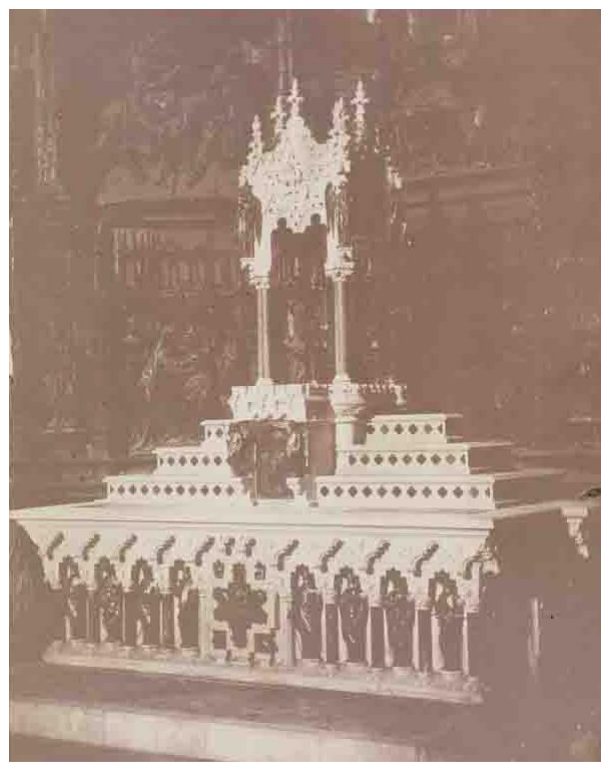

Fig. 9.- Detalle alzado del tabernáculo de la catedral de Oviedo, con fecha [1863 - 1869]. Arquitecto Juan de Madrazo. Museo del Prado, D09021.
Es evidente que en el diseño del altar y tabernáculo, Juan de Madrazo recogió la influencia del arquitecto francés Violletle-Duc, y el gusto de este por las recreaciones de piezas medievales que servían para amueblar los edificios de estilo gótico. Es en este caso cuando Juan de Madrazo, como se podido comprobar anteriormente, demuestra tras su visita para ver el retablo, la gran habilidad y el saber, en el estilo neogótico demostrando la unidad de estilo entre lo existente y lo posteriormente ejecutado. Pese a todo, la obra de Madrazo no reproduce de forma mecánica un modelo gótico concreto, sino que por el contrario presenta la combinación de elementos de carácter historicista y una estilización formal que conforman las características del estilo neogótico.

\section{Traslación de la Catedral al Seminario.}

La obra de Juan de Madrazo permaneció en el presbiterio de la catedral de Oviedo hasta el año 1951, año en que el Sr. Obispo decidió eliminarlo de la catedral sin tener la aprobación del cabildo, cuando fue trasladado al templo del nuevo Seminario seguramente para ennoblecer la capilla mayor de este. En ese momento, para justificar de algún modo el traslado de este altar y tabernáculo se emite un justificante de las obras de la catedral en que se decide hacerlo ya que "se trata de dar mayor explendor al culto", una justificación excesivamente escasa y algo que el cabildo no se creyó en absoluto; en este mismo informe se dice que se sustituye el altar por otro en el centro del crucero, sin gradas encima ni templete que impidan a los capitulares la vista del celebrante y sus ministros, como sucedía con el altar de Madrazo. Aunque en este mismo informe en el que fue aprobado el nuevo retablo, el de Madrazo sería desmontado con el mayor cuidado para poderlo emplazar en el mismo o en otro lugar de la Basílica, algo que finalmente, no se produjo. La mudanza 
de este altar y tabernáculo no contó con la aprobación del cabildo y fue una decisión personal del prelado Lauzurica y Torralba, lo que produjo protestas entre algunos capitulares molestos ya que consideraban que más que un depósito a corto o largo plazo, era una donación irregular, lo que hacía creer que el altar y tabernáculo nunca volverían al lugar que le correspondía en la catedral de Oviedo.

El 11 de diciembre de 1951 el canónigo Arturo Álvarez González, dignidad de chantre, formuló un voto particular sobre el traslado del altar y tabernáculo de Juan de Madrazo en el que muestra su entera disconformidad en estos términos:

...formula el voto particular pidiendo que el altar mayor de nuestra catedral, arrancado y llevado al Seminario, sin previa autorización de este cabildo, sea devuelto y repuesto en el lugar en que siempre estuvo, durante un siglo, por pedido así la voz del pueblo y oponiéndose a que quede allí como depósito, fórmula ingeniosa de evadir una responsabilidad de difícil, por no decir imposible reintegración, una vez emplazado en aquella iglesia...9.

$\mathrm{Al}$ trasladarse al Seminario Ovetense en un principio permanece en el altar completo casi adosado a la pared del ábside, dejando únicamente un pasillo trasero. Desgraciadamente no aparece completo mucho tiempo, ya que tras la reforma litúrgica del Concilio Vaticano II, siendo arzobispo D. Vicente Enrique y Tarancón, se despiezó mutilando casi todas las partes del tabernáculo, dejando exclusivamente el altar intacto y dejando la parte trasera en obra vista de tabiquería. El altar ya llegó al seminario sin dos apóstoles en los laterales ${ }^{10}$ y al desmembrarse el tabernáculo se perdieron otros dos ángeles músicos y los dos ángeles guardianes de los que solo se tiene constancia de su existencia debido a las fotografías que se encuentran del altar y tabernáculo en su forma original en el presbiterio de la catedral de Oviedo, así como en los bocetos que se encuen-

\footnotetext{
${ }^{9}$ Carpeta 110, papeles sueltos, entre los que destaca la obra de la catedral de Oviedo a modo de informe y el voto particular de Arturo Álvarez González en 1951, ACO.

${ }^{10}$ Según el archivero de la catedral D. Agustín cuenta la historia que algún miembro del cabildo que no estaba de acuerdo con la salida del altar y tabernáculo de la catedral escondieron las dos figuras de apóstoles en un jardín que se encontraba anexo a la propia catedral, pero que por la densidad de la hierba no se veían, pero con ello no consiguieron la salida del altar y tabernáculo de la catedral y finalmente no se sabe el motivo real por las que nunca llegaron al seminario y después de tiempo se perdieron, sin que en la actualidad se sepa la ubicación de ellas.
} 
tran en el archivo personal de Juan de Madrazo. Las piezas del expositor y gradas de candelabros se almacenaron en la cripta de la Iglesia Mayor del seminario, el sagrario pasó a la capilla lateral, y fué identificado en mayo de 2010 gracias al aniversario de los 60 años del seminario en el cual se hizo una "limpieza" y se encontró la caja que desgraciadamente estaba destrozada por la oxidación pero con la puerta en muy buen estado de conservación en la que destaca la Cruz de los Ángeles que ya se ha hablado de ella anteriormente. Además de la solicitud que se planteo para la devolución del altar y tabernáculo a la catedral en 1951, se formularon otras dos peticiones más. Siendo rector del seminario José María Hevia, el entonces Deán de la catedral Rafael Somoano solicitó de palabra en 1990 la devolución del altar la cuál se rechazó y se volvió a insistir en el año 2005, a través del canónigo Fabriquero, el arzobispo Benito Gallego y el obispo Carlos Osoro solicitan de palabra un informe al entonces canónigo encargado del patrimonio de la catedral José María Hevia, sobrre la conveniencia o no de la devolución del altar del seminario a su lugar de origen, lo que este desaconseja por no ser factible en el presbiterio de la catedral mantener la unidad de las piezas del altar y estar este sin decoración alguna en su parte trasera. En ninguno de los casos se realizó una solicitud formal ${ }^{11}$.

Actualmente el altar se encuentra íntegro en el presbiterio de la capilla mayor del seminario, exceptuando las dos figuras de los apóstoles que se nombraron anteriormente. En cambio el tabernáculo aparece repartido por la capilla de los seminaristas y el museo de la catedral de Oviedo.

En la capilla de los seminaristas se encuentran las cuatro columnillas de mármol, con las que han realizado un nuevo altar para dicha capilla y un ensamblaje de piezas diferentes entre las que se encuentran un ángel músico y la parte de cúpula mocárabe del tabernáculo con las que se confeccionaron dos ambones diferentes en el taller de restauración tríptico. Uno de estos ambones fue cedido al museo de la catedral de Oviedo, para que en la catedral tuviese a modo de recuerdo un elemento de este altar y tabernáculo del arquitecto Juan de Madrazo del siglo XIX, para el cual se realizó.

Como se ve en las fotografías ambos ambones son idénticos y todos los materiales empleados a excepción de los elementos que se utilizan como base cua-

\footnotetext{
${ }^{11}$ Esta historia ha sido relatada por D. José María Hevia antiguo rector del Seminario Ovetense el cual estuvo presente en el momento de su llegada del altar y tabernáculo al seminario.
} 
drada para la sujeción de la parte superior de los ángeles, las columnas y el elemento superior de cúpula mocárabe forman parte del altar y tabernáculo.

La parte de las escalinatas que conformaban, junto con el sagrario, la parte media del tabernáculo y que apoyaba en el altar, se encuentran en la cripta de la Iglesia Mayor, desmontados y despiezados los trozos en mal estado de conservación y con un deterioro notorio en todos sus elementos.

En el presbiterio de la Catedral de Oviedo se ubica, oculto bajo una alfombra roja, una gran cruz rodeada de un cuadrado que ocupa el contorno exacto donde se encontraba ubicado el altar mayor y tabernáculo de Juan de Madrazo, donde se puede observar en el medio de la cruz el año MCMLII (1952), año en que fue trasladado el altar y tabernáculo al Seminario Ovetense.

El desmontaje y la ubicación en diferentes puntos de partes del altar y tabernáculo así como la pérdida parcial de elementos importantes irremplazables no volverán a permitir que el altar y tabernáculo como lo conformo Juan de Madrazo vuelva a existir.

\section{Conclusiones.}

A Juan de Madrazo le interesaba la arquitectura, el urbanismo y el mobiliario litúrgico para los edificios de estilo gótico. En sus propuestas de mobiliario se observa la misma madurez y seriedad que en otros estudios relacionados con los edificios.

En la propuesta realizada para la catedral de Oviedo de un altar y tabernáculo, se observa claramente el trabajo arquitectónico y a su vez la gran sensibilidad artística que Madrazo poseía. Recogió la influencia del arquitecto Viollet-le-Duc, y el gusto por desarrollar recreaciones de piezas que servían para amueblar los edificios de estilo gótico, sin alterar el entorno que rodeaban, logrando una concordancia entre el edificio y la pieza.

Madrazo en este caso demuestra la maestría propia de un arquitecto restaurador y de su habilidad por manejar la pequeña escala, demostrando así su gusto por el estilo gótico. 


\section{Bibliografía.}

AlcoleA, Rubén, ColominA, Beatriz, et al. (2011). Los viajes de los arquitectos: construir, viajar y pensar. Editado por Craig Buckley y Pollyana Rhee, Universidad de Navarra, 2011.

BENEVOlO, Leonardo (1994). Introducción a la Arquitectura. "La arquitectura gótica" pp. 127-162. Celeste Ediciones, Madrid.

BOSSEGO DE MUSTÉ, Buenaventura (1944). Racionalismo a ultranza en la arquitectura ojival. Memorias de la Real Academia de Ciencias y Artes de Barcelona. Barcelona.

CABELLO y LAPIEDRA, Luis Ma . Arquitectura de las artes decoración arte moderno y construcción. D. Juan de Madrazo, 5 de marzo de 1900. Madrid. Núm. 73

DE LA MADRID, Vidal (1999). La catedral de Oviedo. Historia y restauración. De Ignacio Días Caneja a José Luis Montagut: El altar y tabernáculo de Juan de Madrazo, pp. 230- 235. Ediciones Nobel.

DE GRACIA, Francisco (1992). Construir en lo construido. La arquitectura como modificación. Editorial Nerea.

DíAZ PÉREZ, Javier (1985). “La exposición de "Los Madrazo", en el museo municipal". Villa en Madrid, Ayuntamiento de Madrid.

DíAZ PÉREZ, Javier (1985). “Los Madrazo y las Exposiciones Nacionales”. Villa de Madrid, Ayuntamiento de Madrid.

GONZÁLEZ VARAS, Ignacio (2005). Conservación de bienes culturales. Teoría, historia, principios y normas, Cátedra, Madrid.

GONZÁLEZ VARAS, Ignacio. “Los nuevos maestros mayores: los arquitectos restauradores de la catedral de León" [en línea]. Artículo. Fecha de consulta: Abril 2015. Disponible en www.saber.es/web/biblioteca/libros/lacatedral-de-leon-el.../g2.pdf.

LAMPÉREZ Y ROMEA, Vicente (1898). “Los trazados geométricos de los monumentos españoles de la Edad Media: Catedral de Burgos, León y Reims". Resumen de arquitectura, 1898.

NAVASCUÉs PAlACIO, Pedro (1985). El arquitecto Juan de Madrazo y Kuntz. Los Madrazo, una familia de artistas. Ayuntamiento de Madrid, Madrid.

NAVASCUÉS PALACIO, PEDRO (1973). Arquitectura y arquitectos madrileños del siglo XIX, Madrid. 
NAVASCUÉs PAlACIO, Pedro (1987). “La creación de la Escuela de Arquitectura" en Historia de la arquitectura del neoclasicismo al modernismo pp. 49-51, Madrid.

Panadero Peropadre, Nieves y Azcárez Ristori, Jose María de (2005). Los estilos medievales en la arquitectura madrileña del siglo XIX (1780-1868). Universidad Complutense de Madrid.

PÉREZ NievA, Alfonso (1895). Un viaje a Asturias pasando por León. Librería de Victorio Suarez, Madrid.

PÉREZ MONZÓN, Olga (2003). Catedrales Góticas: catedrales de España. Ediciones Jaguar, Madrid.

PÉREZ GIL, Javier (2013). “Los Torbado: El patrimonio leonés y la evolución de la restauración arquitectónica en España". Revista de Historia del Arte.

PRIETO GONZÁLEZ, Jose Manuel. "La escuela de arquitectura y de enseñanza de la restauración monumental" [en línea]. Artículo. Fecha de consulta: Abril 2015. Disponible en www.saber.es/web/biblioteca/libros/la-catedralde-leon-el.../g2.pdf.

REVISTA de la arquitectura nacional y extranjera: órgano de la sociedad central de arquitectos. Editor Sociedad Central de Arquitectos. 1878.

RIVERA BlANCO, Javier (2000). Principios de la restauración en la nueva Europa. Conferencia internacional de conservación del patrimonio. Fundación del patrimonio histórico de Castilla y León. Valladolid.

Rivera Blanco, J. Altés, J. GonZÁlez Fraile, J. R. SOlA (1999). Patrimonio, restauración y nuevas tecnologías. Instituto Español de Arquitectura. Valladolid.

Viollet-Le-Duc. Caractére de l'architecture gothique. Gazette des architectes et du bátiment, extrait de XIII éme des Entretiens sur l'architecture. Paris 1868-1869.

Viollet-Le-Duc, Eugéne E. et LAssus, Jean - Baptiste. Monographie de Notre Dame de Paris et de la nouvelle sacristie de MM. Lassus et Viollet-le-Duc précédée d'une notice historique et archeologique par Celtibére. Paris, 1856. 